ARTICLE

Received 23 Sep 2013 | Accepted 18 Feb 2014 | Published 11 Mar $2014 \quad$ DOl: 10.1038/ncomms4466

\title{
Ultrafast angular momentum transfer in multisublattice ferrimagnets
}

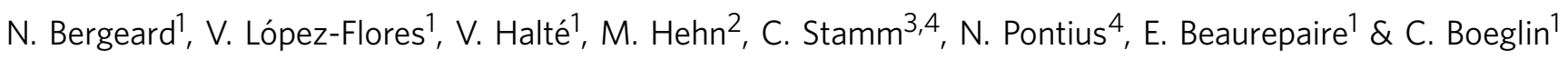

Femtosecond laser pulses can be used to induce ultrafast changes of the magnetization in magnetic materials. However, one of the unsolved questions is that of conservation of the total angular momentum during the ultrafast demagnetization. Here we report the ultrafast transfer of angular momentum during the first hundred femtoseconds in ferrimagnetic $\mathrm{Co}_{0.8} \mathrm{Gd}_{0.2}$ and $\mathrm{Co}_{0.74} \mathrm{~Tb}_{0.26}$ films. Using time-resolved $\mathrm{X}$-ray magnetic circular dichroism allowed for time-resolved determination of spin and orbital momenta for each element. We report an ultrafast quenching of the magnetocrystalline anisotropy and show that at early times the demagnetization in ferrimagnetic alloys is driven by the local transfer of angular momenta between the two exchange-coupled sublattices while the total angular momentum stays constant. In $\mathrm{Co}_{0.74} \mathrm{~Tb}_{0.26}$ we have observed a transfer of the total angular momentum to an external bath, which is delayed by $\sim 150 \mathrm{fs}$.

\footnotetext{
${ }^{1}$ Institut de Physique et de Chimie des Matériaux de Strasbourg, UMR7504, CNRS et Université de Strasbourg, 67034 Strasbourg, France. ${ }^{2}$ Institut Jean Lamour, Université Henri Poincaré, UMR 7198, 54506 Nancy, France. ${ }^{3}$ Department of Materials, ETH Zürich, CH-8093 Zürich, Switzerland. ${ }^{4}$ Institut für Methoden und Instrumentierung der Forschung mit Synchrotronstrahlung Helmholtz-Zentrum Berlin für Materialien und Energie GmbH, Albert-Einstein Strasse 15, 12489 Berlin, Germany. Correspondence and requests for materials should be addressed to C.B. (email: christine.boeglin@ipcms.unistra.fr).
} 
T he mechanism of ultrafast quenching of the magnetization triggered by a femtosecond laser pulse is currently still hotly debated. Despite the large amount of work devoted to the characterization of femtosecond demagnetization in various metallic systems ${ }^{1-11}$, so far no consensus over the sub-picosecond demagnetization mechanisms has emerged. The basic phenomena involved, especially regarding the dissipation of angular momenta, are still disputed ${ }^{2-9,12}$. Different other aspects are still discussed controversially, especially the timescales characterizing energy and angular momentum transfer between various degrees of freedom, such as electrons (e), spins (s) and phonons (ph). However, the literature documents critical differences between rare earth (RE) elements ${ }^{4}$ and transition metals (TMs) $3,5,7,8,10,11$. The disparities lie in the couplings between $\mathrm{e}, \mathrm{s}$, and $\mathrm{ph}$, and are related to the localization and hybridization of the electron orbitals carrying the spin momentum. As the loss of magnetization requires transfer of energy into the spin system and of angular momentum out of it, one of the important and unsolved questions is related to the description of the fundamental processes involving the transfer of angular momentum out of $s$.

In earlier work, it has been proposed that phonon- or defectmediated spin-flip scattering ${ }^{5}$ or electron-magnon spin-flip scattering ${ }^{11}$ can account for the transfer of angular momentum on the femtosecond timescale but recent quantitative $a b$ inito calculations show that the contribution of electron-phonon spin-flip scattering is too small to describe the experimental femtosecond demagnetization ${ }^{13}$. Alternatively, ultrafast quenching of the magnetocrystalline anisotropy (MCA) has been proposed by Boeglin et al. ${ }^{10}$ as a new mechanism of demagnetization in TM and has been supported by microscopic theoretical work ${ }^{6}$. Recently, a substitute for the transfer of angular momentum during the ultrafast demagnetization has been proposed ${ }^{14-16}$ involving ultrafast superdiffusive spin-polarized transport. In this process, no angular momentum transfer to the lattice is required. However, the relative weights of each pre-cited contribution to the total demagnetization process are still unclear ${ }^{17-20}$.

In the case of multi-lattice magnets, the situation is more complex $^{21}$ since additional mechanisms may emerge. For instance, transfer of angular momentum between magnetic lattices has been predicted by Mentink et al. ${ }^{22}$ and ab initio density functional theory (DFT) calculations performed by Wienholdt et al. ${ }^{12}$ have demonstrated that the transfer of angular momentum between both sublattices is a key phenomenon to establish a transient ferromagnetic-like state in FeCoGd alloys ${ }^{23}$. An experimental attempt to demonstrate the efficiency of such a process has been made by Graves et al. ${ }^{24}$ using time-resolved resonant X-ray scattering in FeCoGd alloys. However, the scenario they propose for the transfer of angular momenta strongly relies on the microscopic inhomogeneity of the sample studied and cannot be viewed as a generic mechanism.

Here, we report experimental results evidencing the initial conservation and the following dissipation of the total angular momentum during the demagnetization time by measuring the angular momentum in a quantitative way in ferrimagnetic TM-RE alloys. We have used time-resolved X-ray magnetic circular dichroism (XMCD) at the $\mathrm{TM} \mathrm{L}_{2,3}$ and $\mathrm{RE} \mathrm{M}_{4,5}$ edges at the BESSY II femtoslicing source of the Helmholtz-Zentrum Berlin ${ }^{7}$. This tool combines element, spin and orbital sensitivity ${ }^{9,25,26}$ with femtosecond time resolution resolving the ultrafast dynamics of the orbital $\left(L^{i}(t)\right)$, spin $\left(S^{i}(t)\right)$ and total angular momentum $\left(J^{i}(t)\right)$ (where $i$ stands for $\mathrm{Co}$, $\mathrm{Gd}$ or $\mathrm{Tb}$ ) in ferrimagnetic $\mathrm{Co}_{0.8} \mathrm{Gd}_{0.2}$ and $\mathrm{Co}_{0.74} \mathrm{~Tb}_{0.26}$ alloys. Our results show on what timescales and through which microscopic mechanism the total angular momentum is conserved in the case of a multisublattice magnet. They reveal that, to conserve the total angular momentum, the ferrimagnetic system involves two compensating angular momenta that flow in opposite directions, allowing for the loss of magnetization in each subsystem during the first $140 \pm 60 \mathrm{fs}$ of demagnetization and resulting in a net loss of the magnetization in the system. We have determined at what delay after the laser excitation the systems start to transfer angular momentum to the external reservoir. Backed by the ultrafast dynamics of the ratio $L^{\mathrm{Co}}(t) / S^{\mathrm{Co}}(t)$, our results reveal an ultrafast quenching of the MCA in both ferrimagnetic films ${ }^{10}$. Finally, we show that the atomic description is still valid for Gd in the first hundred femtoseconds after the laser excitation, because the orbital momentum $L^{\mathrm{Gd}}(t)=0$ at all times.

\section{Results}

Experimental details. The magnetic configuration at the thermodynamic equilibrium of the two different ferrimagnetic alloy films $\mathrm{Co}_{0.8} \mathrm{Gd}_{0.2}$ and $\mathrm{Co}_{0.74} \mathrm{~Tb}_{0.26}$ have been analysed by $\mathrm{XMCD}$ measurements; see Methods. Thanks to the chemical sensitivity of XMCD, our previous static measurements ${ }^{27}$ are used to quantify the ultrafast dynamics of the angular momenta $S_{\mathrm{z}}^{i}(t), L_{\mathrm{z}}^{i}(t)$ and $J_{\mathrm{z}}^{i}$ $(t)$ in Co and in the RE elements. To test the possible ultrafast increase in $L^{i}(t)$ starting from an initial value $L^{i}(t<0)=0$, we study one of the most convenient cases, $\mathrm{Gd}$ in $\mathrm{Co}_{0.8} \mathrm{Gd}_{0.2}$. In contrast, the $\mathrm{RE} \mathrm{Tb}$ has a large orbital momentum $(L=3)$ and is therefore well suited to study the demagnetization dynamics of the orbital momenta ${ }^{27}$. According to element-resolved hysteresis loops obtained for both alloys ${ }^{29}$, we conclude that our films are homogenous and are fully saturated under the applied magnetic field of $5 \mathrm{kOe}$. Therefore, we have $L(t) \sim L_{\mathrm{z}}(t)$ and $S(t) \sim S_{\mathrm{z}}(t)$ for Co, Gd and Tb. Thus, $L(t)$ and $S(t)$ are aligned parallel, so that we may define the time-dependent angular momentum $J^{i}(t)=L^{i}(t)+S^{i}(t)$ per element $i$.

The X-ray transmission experiment, using an infrared laser pump and X-ray probe configuration, is schematically shown in Fig. 1. The external magnetic field is applied along the
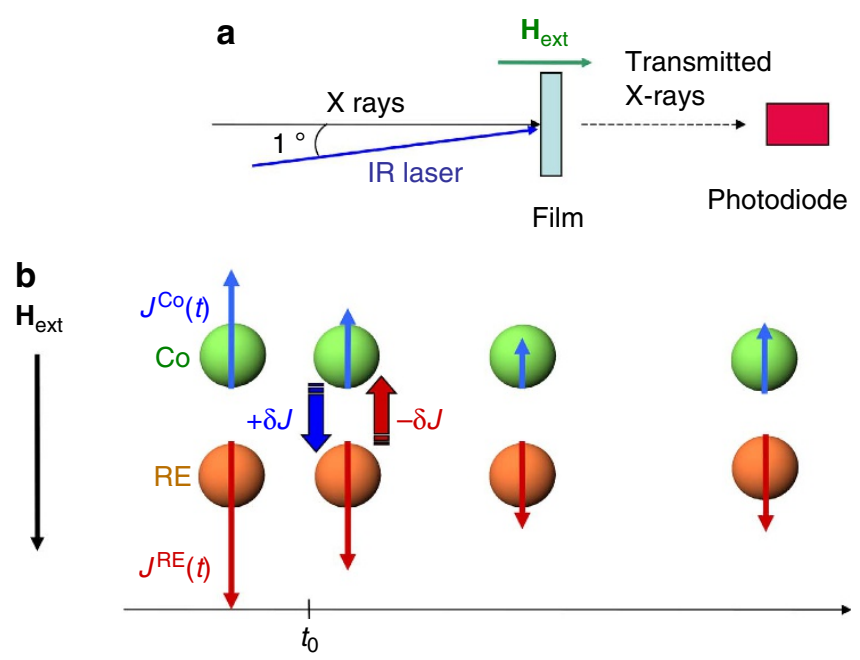

Figure 1 | Experimental set-up and the microscopic model. (a) Scheme of the X-ray transmission experiment using an infrared laser pump and X-ray probe configuration. The external magnetic field is applied parallel to the incoming X-rays. (b) Sketch of the time evolution of the pump-induced atomic magnetization dynamics on the exchange-coupled Co and RE in the ferrimagnetic multisublattice. Ultrafast dynamics of $J^{\prime}(t)$ for each element is illustrated and start at the temporal overlap $t_{0}=0$. At any time, both elements are exchange coupled and show antiferromagnetic order, attesting that we do not cross the compensation temperature $T_{\text {comp }}$. During the demagnetization, a coupling between the two angular momenta may exist in the ferrimagnet. 
propagation direction of the X-rays. The lower part sketches the time evolution of the pump-induced atomic demagnetization on the exchange-coupled $\mathrm{Co}$ and $\mathrm{RE}$ in the multisublattice ferrimagnet. The time $t_{0}=0$ is defined by the temporal overlap between the laser pulse and the X-ray pulse. A film thickness of $15 \mathrm{~nm}$ ensures a homogenous infrared laser excitation. The incident X-rays are circularly polarized and time-resolved XMCD is measured using the difference of intensities measured in transmission between two opposite magnetic fields $+\mathbf{H}$ and $-\mathbf{H}$ applied parallel to the X-rays (see Methods).

Ultrafast demagnetization in CoGd. In Fig. 2a we show the pump-probe results obtained at the $\mathrm{Co}_{2,3}$ and $\mathrm{Gd} \mathrm{M}_{4,5}$ edges for the $\mathrm{Co}_{0.8} \mathrm{Gd}_{0.2}$ alloy film. The normalized XMCD values at negative time delays match the XMCD magnitudes recorded at the Co $\mathrm{L}_{3}, \mathrm{Co}_{2}, \mathrm{Gd} \mathrm{M}_{5}$ and $\mathrm{Gd} \mathrm{M}_{4}$ edges during quantitative static XMCD measurements ${ }^{27}$. Applying the sum rules for the Co $\mathrm{L}_{2,3}$ and for the $\mathrm{Gd} \mathrm{M}_{4,5}$ edges $9,25,26$, we extract the ultrafast dynamics of the spin momentum $S^{i}(t)$ (black circles) and the orbital momentum $L^{i}(t)$ (red circles) for Co and for Gd. Quantitative data for the time-dependent values of $S^{i}(t)$ and $L^{i}(t)$ are displayed in units of $\hbar$ per atom in Fig. 2b,c (red and black symbols). The values at negative delays are normalized to the values measured at thermodynamic equilibrium ${ }^{27}$. The continuous lines are the results of the simulations of $S^{i}(t)$ (black line) and $L^{i}(t)$ (blue line; see Methods), whereas the green line is the ratio of these results.

Analysing the time-dependent spin and orbital momenta for Co (Fig. 2b) we observe that, similar to CoPd films ${ }^{10}$, the magnitude of the demagnetization observed for $S^{\mathrm{Co}}(t)$ and $L^{\mathrm{Co}}(t)$ are different leading to a large quenching of the ratio
$L^{\mathrm{Co}}(t) / S^{\mathrm{Co}}(t)$ before $t=1 \mathrm{ps}$ (Fig. 2a, green symbols). The thermalization times of $S^{\mathrm{Co}}(t)$ and $L^{\mathrm{Co}}(t)$ are identical $\left(\tau_{\text {therm }}=200 \pm 20 \mathrm{fs}\right)$ within the error bars. For Gd (Fig. 2c), we notice a large decrease of $S^{\mathrm{Gd}}(t)$ while $L^{\mathrm{Gd}}(t) \sim 0$. The dynamics of $S^{\mathrm{Gd}}(t)$ show that the thermalization time is much longer, $\tau_{\text {th }}=480 \pm 40 \mathrm{fs}$ (ref. 27). Interestingly, the value $L^{\mathrm{Gd}}(t)=0$ stays constant over this time, although the orbital momentum was previously assumed to be an angular momentum sink to explain the loss of spin momentum on the sub-picosecond timescale. The excess of angular momentum stored in the electron orbit was assumed to be transferred to the lattice only at longer times $(\sim 1 \mathrm{ps})^{28}$. A transfer to the lattice was also proposed by Stamm et al. ${ }^{7}$ in the case of $\mathrm{Ni}$, although on the faster subpicosecond timescale. The quantitative ultrafast dynamics of $S^{\mathrm{Gd}}(t)$ and $L^{\mathrm{Gd}}(t)$ reported here excludes any ultrafast transfer from the $4 f$ spin angular momentum towards the orbital angular momentum in Gd. In addition, we rule out any ultrafast transfer from Co towards the Gd orbital momentum.

Ultrafast demagnetization in CoTb. For the $\mathrm{Co}_{0.74} \mathrm{~Tb}_{0.26}$ alloy the excited-state temperature is below $T_{\text {comp }}$, thus leading to faster demagnetization rates for the $\mathrm{RE}\left(\tau_{\text {therm }}=280 \pm 40 \mathrm{fs}\right)$ as compared with other experiments where the excited-state temperature is in the vicinity of $T_{\mathrm{C}}$ (refs 23,27 ). The pump-probe results measured at the $\mathrm{Co}_{2,3}$ and $\mathrm{Tb}_{4,5}$ edges are shown in Fig. $3 \mathrm{a}$ and transformed into ultrafast dynamics of $S^{i}(t)$ and $L^{i}(t)$ for $\mathrm{Co}$ and $\mathrm{Tb}$ as shown in Fig. 3b,c (red and black symbols). The normalized XMCD values at negative time delays match the XMCD magnitude recorded at the $\mathrm{Co}_{3}$, Co $\mathrm{L}_{2}, \mathrm{~Tb}_{5}$ and $\mathrm{Tb}$ $\mathrm{M}_{4}$ edges during quantitative static XMCD measurements ${ }^{27}$. Applying the sum rules, we can extract the ultrafast dynamics of a

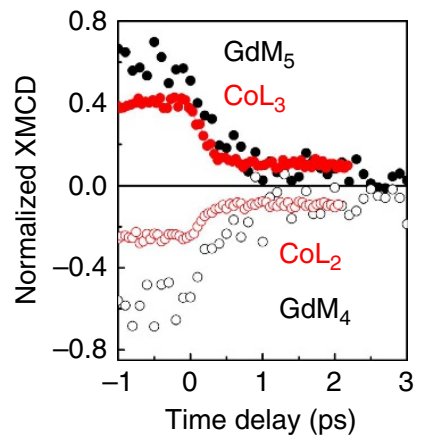

b

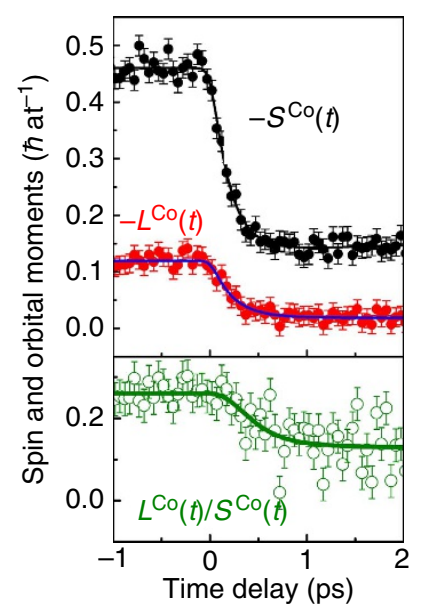

C

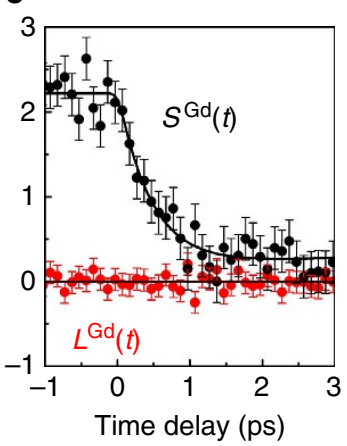

Figure 2 | Dynamics of spin and orbital momenta for $\mathbf{C o}$ and $\mathbf{G d}$ in $\mathbf{C o}_{\mathbf{0}, \mathbf{8}} \mathbf{G} \mathbf{G d}_{\mathbf{0 . 2}}$. (a) Time-resolved XMCD at the $\mathrm{Co}_{L_{3}}$ (filled red circles), Co $L_{2}$ (empty red circles), $\mathrm{Gd} \mathrm{M}_{5}$ (filled black circles) and $\mathrm{Gd} \mathrm{M}_{4}$ edges (empty black circles) measured on a $15-\mathrm{nm} \mathrm{Co}_{0,8} \mathrm{Gd}_{0.2}$ alloy film. The XMCD values at negative time delays match the XMCD magnitude recorded at the $\mathrm{Co}_{3}, \mathrm{Co}_{2}, \mathrm{Gd} \mathrm{M}_{5}$ and $\mathrm{Gd} \mathrm{M}_{4}$ edges during quantitative static XMCD measurements ${ }^{27}$. The pump fluence used during our experiment was adjusted to $8 \mathrm{~mJ} \mathrm{~cm}^{-2}$. (b) Ultrafast dynamics of the spin and orbital momenta $-S^{\mathrm{Co}}(t)$ (black circles) and $-L^{\mathrm{Co}}(t)$ (red circles) for $\mathrm{Co}_{0}$ as extracted by applying the sum rules. The values of $L^{\mathrm{Co}}(t)$ and $S^{\mathrm{Co}}(t)$ at negative delays are normalized to the static values $L^{\mathrm{Co}}$ and $S^{\mathrm{Co}}$ at thermodynamic equilibrium ${ }^{27}$. The continuous lines are the simulations of $S^{\mathrm{Co}}(t)$ (black line) and $L^{\mathrm{Co}}(t)$ (blue line; see Methods) with characteristic thermalization times of $\tau^{\mathrm{Co}_{\mathrm{o}}}$ therm $=200 \pm 20 \mathrm{fs}$ for both momenta. The ratio $L^{\mathrm{Co}}(t) / S^{\mathrm{Co}}(t)$ (green circle) obtained as a function of the delay time shows that the orbital momentum reduces more than the spin momentum during the ultrafast demagnetization. (c) Ultrafast dynamics of the spin and orbital momenta $S^{\mathrm{Gd}}(t)$ (black circles) and $L^{\mathrm{Gd}}(t)$ (red circles) for $\mathrm{Gd}$. The values at negative delays are normalized to the static values of $L^{\mathrm{Gd}}$ and $S^{\mathrm{Gd}}$ provided by the static XMCD measurements ${ }^{27}$. A constant value of $L^{\mathrm{Gd}}(t)=0$ is evidenced during the ultrafast demagnetization process. The continuous line is a simulation of $S^{\mathrm{Gd}}(t)$ (black line) with a characteristic thermalization time of $\tau \mathrm{Gd}_{\text {therm }}=480 \pm 40 \mathrm{fs}$. The simulations of $L^{i}(t)$ and $S^{i}(t)$ (where $i$ stands for $\mathrm{Co}, \mathrm{Gd}$ or Tb) have been obtained using a nonlinear least square fitting procedure. The extracted error bar on $\tau_{\text {therm }}^{i}$ is the s.d., which is given as an output parameter of the fitting procedure. The error bars obtained for $L^{i}(t)$ and $S^{i}(t)$ as shown in $\mathbf{b}$ and $\mathbf{c}$ are given by the s.d. of the experimental data with respect to the fitting functions (see Methods). 
a

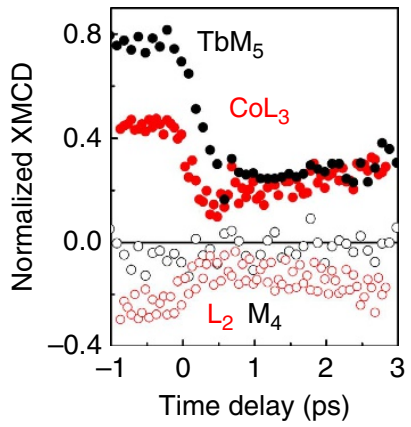

b

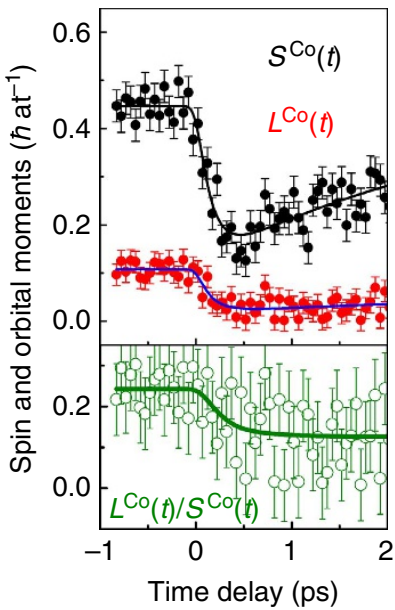

C

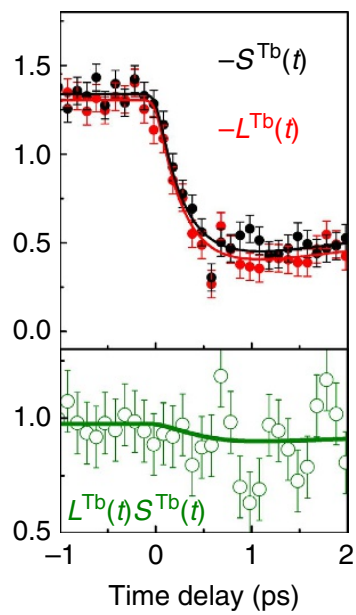

Figure 3 | Dynamics of spin and orbital momenta for $\mathbf{C o}$ and $\mathbf{T b}$ in $\mathbf{C o}_{\mathbf{0}, \mathbf{7 4}} \mathbf{T} \mathbf{b}_{\mathbf{0}, \mathbf{2 6}}$ (a) Time-resolved XMCD at the Co $L_{3}$ (filled red circles), Co $L_{2}$ (empty red circles), Tb $M_{5}$ (filled black circles) and Tb $M_{4}$ edges (empty black circles) measured on a 15-nm $\mathrm{Co}_{0,74} \mathrm{~Tb}_{0,26}$ alloy film. The XMCD values at negative time delays match the XMCD magnitude recorded at the $C_{0} L_{3}, C_{0} L_{2}, T b M_{5}$ and $T b M_{4}$ edges during quantitative static XMCD measurements ${ }^{27}$. The pump fluence used during our experiment was adjusted to $12 \mathrm{~mJ} \mathrm{~cm}^{-2}$. (b) Ultrafast dynamics of the spin and orbital momenta $S^{\mathrm{Co}}(t)($ black circles) and $L^{\mathrm{Co}}(t)$ (red circles) for Co extracted by applying the sum rules. The continuous lines are the simulations of $S^{\mathrm{Co}}(t)$ (black line) and $L^{\mathrm{Co}}(t)$ (blue line; see Methods) with characteristic thermalization times of $\tau^{\mathrm{Co}}$ therm $=180 \pm 40 \mathrm{fs}$ for both momenta. The dynamics of the ratio $L^{\mathrm{Co}}(t) / S^{\mathrm{Co}}(t)$ (green circle) shows that the orbital momentum decays more than the spin momentum during the ultrafast demagnetization. (c) Ultrafast dynamics of the spin and orbital momenta $-S^{\mathrm{Tb}}(t)$ (black circles) and $-L^{\mathrm{Tb}}(t)$ (red circles) for Tb. The continuous lines are the simulations of $S^{\mathrm{Tb}}(t)$ (black line) and $L^{\mathrm{Tb}}(t)$ (red line; see Methods) with characteristic thermalization times of $\tau^{\mathrm{Tb}}$ therm $=280 \pm 40 \mathrm{fs}$ for both momenta. The simulations of $L^{i}(t)$ and $S^{i}(t)$ have been obtained using a nonlinear least square fitting procedure. The extracted error bar on $\tau_{\text {therm }}^{i}$ is the s.d., which is given as an output parameter of the fitting procedure. The error bars obtained for $L^{i}(t)$ and $S^{i}(t)$ as shown in $\mathbf{b}$ and $\mathbf{c}$ are given by the s.d. of the experimental data with respect to the fitting functions (see Methods).

the spin and orbital momenta $S^{i}(t)$ (black circles) and $L^{i}(t)$ (red circles) for Co and for Tb. For Co and Gd we can neglect the magnetic dipole term $T_{\mathrm{z}}(t)$ so that the spin momentum is then defined by $S(t)=S^{\text {eff }^{2}}(t)^{27}$. For Tb, according to atomic calculations done by Teramura et al. ${ }^{29}$, we take $T_{\mathrm{z}} / S=-0.08$. We further correct the sum rule-extracted value $S^{\text {eff }}(t)$ by a value $T_{\mathrm{z}}(t)^{27}$ representing a time constant proportion of $8 \%$ of $S(t)$. This assumption leads to an underestimation of $8 \%$ of $S^{\mathrm{Tb}}(t)$ for $t>0$ assuming a virtual and complete quenching of $T_{\mathrm{z}}(t)$ by the pump laser at $t>0$. The values at negative delays are normalized to the values measured at thermodynamic equilibrium ${ }^{27}$. The continuous lines correspond to the simulations of $S^{i}(t)$ (black line) and $L^{i}(t)$ (blue line; see Methods) while the green lines are the ratios between these numerical results. In Fig. 3b,c we observe an ultrafast quenching of $S^{i}(t)$ and $L^{i}(t)$ for Co and Tb. For each element, the thermalization times of $S^{i}(t)$ and $L^{i}(t)$ are identical (Co $\tau_{\text {th }}=180 \pm 40 \mathrm{fs}$ and $\mathrm{Tb} \tau_{\text {th }}=280 \pm 30 \mathrm{fs}$ ). Computing the ratio $L^{i}(t) / S^{i}(t)$ reveals an ultrafast quenching for Co but for $\mathrm{Tb}$ any change is smaller than the error bars (Fig. 3c, green symbols).

The ultrafast quenching of $L^{\mathrm{Co}}(t) / S^{\mathrm{Co}}(t)$ in $\mathrm{Co}_{0.8} \mathrm{Gd}_{0.2}$ and $\mathrm{Co}_{0.74} \mathrm{~Tb}_{0.26}$ leads to several conclusions. Interestingly, one notices that in this experiment we quenched $L^{\mathrm{Co}}(t) / S^{\mathrm{Co}}(t)$ towards the value of 0.12 , which is the value of hexagonal close packed Co (ref. 9). This reflects a transition from the highly anisotropic electronic distribution in Co-RE alloys towards the more isotropic distribution in hexagonal close packed structures. The quenching of $L^{\mathrm{Co}}(t) / S^{\mathrm{Co}}(t)$ is in agreement with the model of ultrafast quenching of MCA proposed by Boeglin et al. ${ }^{10}$, in the framework of Bruno's model ${ }^{30}$. This model predicts a direct relationship between the anisotropy of the orbital momentum and the magnetic anisotropy energy in $3 d$ TMs. In the case of $4 f$ localized moments, the simple relationship given by Bruno ${ }^{30}$ is no longer valid. Considering this limitation for RE $4 f$ moments, the ultrafast dynamics of $L(t) / S(t)$ does not reflect the quenching of the MCA. However, the ultrafast dynamics of $L^{\mathrm{Tb}}(t) / S^{\mathrm{Tb}}(t)$ observed in Fig. $3 c$ (green line) is consistent with the fact that the $4 f$ moments are not directly pumped by the infrared laser but are exchange coupled with the laser pumped $3 d-5 d$ states, explaining a quasi-simultaneous demagnetization of $L^{\mathrm{Tb}}(t)$ and $S^{\mathrm{Tb}}(t)$, whereas the $3 d$ moments show an ultrafast quenching of $L^{\mathrm{Co}}(t) / S^{\mathrm{Co}}(t)$. Considering the low infrared pump energy, we suggest that the exchange coupling between the $4 f$ and the pumped $5 d 3 d$ moments prevent large ultrafast quenching of the magnetocrystalline and dipolar anisotropy of the $4 f$ moments. It follows that under the given pump conditions, the proposed correction value of $T_{\mathrm{z}}(t)$ as a constant proportion of $S(t)$ is justified for $\mathrm{Tb}$.

Total angular momentum. We calculate the angular momentum $f^{i}(t)=L^{i}(t)+S^{i}(t)$ by adding the element-resolved $L^{i}(t)$ and $S^{i}(t)$. We multiply $f^{i}(t)$ by the elemental concentration in the alloy for each $i=\mathrm{Co}$, Gd or $\mathrm{Tb}$ in $\mathrm{Co}_{0.8} \mathrm{Gd}_{0.2}$ (Fig. 4a) and $\mathrm{Co}_{0.74} \mathrm{~Tb}_{0.26}$ (Fig. 4b). All individual angular momenta $f^{i}(t)$ are quenched on laser excitation at $t=0 \mathrm{ps}$. For each alloy, we then derive the total angular momentum $J(t)=J^{\mathrm{Co}}(t)+J^{\mathrm{Gd}}(t)$ (Fig. 4a, blue open symbols) and $J(t)=J^{\mathrm{Co}}(t)+J^{\mathrm{Tb}}(t)$ (Fig. 4b, blue open symbols). To account for the time dependence of $T_{\mathrm{z}}(t)$ in $\mathrm{Tb}$, we estimated the variation for $J(t)$ to be $\sim 0.020 \hbar$ at ${ }^{-1} \%$, less than the given error bars of $\pm 0.035 \hbar$ at $^{-1} \%$. In $\mathrm{Co}_{0.8} \mathrm{Gd}_{0.2}$, where the working temperature is close to but above $T_{\text {comp }}$, we have $J^{\mathrm{Co}} \approx-J^{\mathrm{Gd}}$ and the total angular momentum is mostly compensated. The remaining $J(t)$ in CoGd is too small compared with the experimental noise level to reliably detect changes within the first few hundred of femtoseconds. The small negative values of $J(t)$ observed for $t>1 \mathrm{ps}$ illustrates the non-equilibrium state of the system determined by the working temperature and pump conditions ${ }^{27}$. 
a

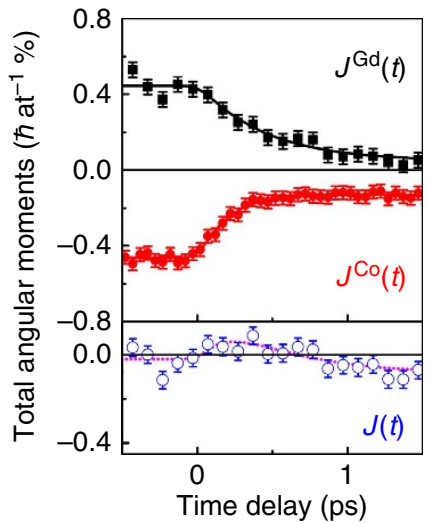

b

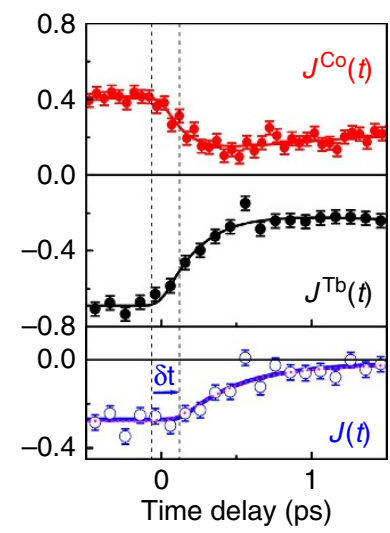

Figure 4 | Ultrafast dynamics of the individual and total angular momenta. (a) Angular momenta $J^{\mathrm{Co}}(t)$ (red circles), $J^{\mathrm{Gd}}(t)$ (black circles) and $J(t)=J^{\mathrm{Co}}(t)+J^{\mathrm{Gd}}(t)$ (blue circles) normalized by the composition (that is, $J^{\mathrm{Co}}(t)=\left(L^{\mathrm{Co}}(t)+S^{\mathrm{Co}}(t)\right) \times 0.80$ and $\left.J^{\mathrm{Gd}}(t)=\left(L^{\mathrm{Gd}}(t)+S^{\mathrm{Gd}}(t)\right) \times 0.2\right)$ in the $\mathrm{Co}_{0,8} \mathrm{Gd}_{0.2}$ film. The continuous lines are simulations of $J^{\mathrm{Co}}(t)$ (red line), $J \mathrm{Gd}(t)$ (black line), whereas the magenta line is the sum of the two simulations $J^{\mathrm{Co}}(t)$ (red line) and $J^{\mathrm{Gd}}(t)$ (black line). (b) Angular momenta $J^{\mathrm{Co}}(t)$ (red circles), $J^{\mathrm{Tb}}(t)$ (black circles) and $J(t)=J^{\mathrm{Co}}(t)+J^{\mathrm{Tb}}(t)$ (blue circles) normalized by the composition (that is, $J^{\mathrm{Co}}(t)=\left(L^{\mathrm{Co}}(t)+\right.$ $\left.S^{\mathrm{Co}}(t)\right) \times 0.74$ and $\left.J^{\mathrm{Tb}}(t)=\left(L^{\mathrm{Tb}}(t)+S^{\mathrm{Tb}}(t)\right) \times 0.26\right)$ measured in $\mathrm{Co}_{74} \mathrm{~Tb}_{26}$ alloy film. The continuous lines are simulations of $J^{\mathrm{Co}}(t)$ (red line), $J^{\mathrm{Tb}}(t)$ (black line) and $J(t)$ (blue line). The sum of both simulations $\left(J^{\mathrm{Co}}(t)+J^{\mathrm{Tb}}(t)\right.$-in magenta) cannot be distinguished from the simulation of $J(t)$ (blue line). The loss of the total angular momentum $J(t)$ is delayed by $\delta t=140 \pm 60 \mathrm{fs}$ compared with the time at which the demagnetization of both sublattices starts. The simulations of $J^{i}(t)$ and $J(t)$ have been obtained using a nonlinear least square fitting procedure. Error bars on $J^{i}(t)$ and $J(t)$ displayed in $\mathbf{a}$ and $\mathbf{b}$ correspond to the s.d. of experimental data with respect to the fitting functions (see Methods).

For $\mathrm{Co}_{0.74} \mathrm{~Tb}_{0.26}$ where the working temperature is below $T_{\text {comp }}$, we have $J^{\mathrm{Co}}<-J^{\mathrm{Tb}}$ and a sizable value of $J(t)=J^{\mathrm{Co}}(t)+$ $J^{\mathrm{Tb}}(t)$ is obtained that allows us to follow unambiguously the ultrafast dynamics of $J(t)$. The validity of the determination of $J(t)$ is supported by the limited error introduced by neglecting a virtual quenching of $T_{\mathrm{z}}(t)$ at $t>0$. In such a case, we estimate that for $J(t)$ we make an error of $0.025 \hbar$ at ${ }^{-1}$, well below the experimental error bars of $0.035 \hbar$ at $^{-1}$ given for the experimental values (Fig. 4b). Analysing the dynamics of $J(t)$, we observe a loss of the total angular momentum $J(t)$ towards an external bath until the value $J(t) \approx 0$ is reached at $t=1.5 \mathrm{ps}$. This dissipation happens with a characteristic time of $300 \pm 50 \mathrm{fs}$ (Fig. $4 \mathrm{~b}$ ). At $t=1.5 \mathrm{ps}$, the system is at quasi-equilibrium at a temperature close to $T_{\text {comp }}{ }^{27}$ and the value $J(t)=0$ shows that the CoTb alloy is now magnetically compensated. The appealing feature is that the quenching of $J(t)$ is delayed by $\delta t=140 \pm 60 \mathrm{fs}$, whereas both $J^{\mathrm{Co}}(t)$ and $J^{\mathrm{Tb}}(t)$ have already started to be quenched (Fig. 4b). The value $\delta t$ is estimated by performing simulations using a double exponential function for $J(t)$ (Fig. $4 \mathrm{~b}$, blue line; see Methods). In Fig. $4 \mathrm{~b}$ we superpose the weighted sum of the two-fit functions obtained for $J^{\mathrm{Co}}(t)$ and $J^{\mathrm{Tb}}(t)$ as the magenta dotted line. The two-fit functions for $J(t)$ agree perfectly. The delay of $\delta t=140 \pm 60 \mathrm{fs}$ is attributed to an ultrafast transfer of angular momenta between the coupled $\mathrm{Co}$ and $\mathrm{Tb}$ sublattices.

\section{Discussion}

Compared with previous work by Medapalli et al. ${ }^{31}$ and Graves et $a .^{24}$, we provide quantitative time-resolved and

element-selective angular momenta $f^{i}(t)$ in ferrimagnetic TMRE alloys. Medapalli et al. ${ }^{31}$ argued that a direct transfer of angular momentum occurs between the TM to the RE sublattices when the temperature of the sample is below the temperature of magnetic compensation. Although a model developed by Mentink et $a l^{22}$ supported their claim, no direct proof of such a mechanism has been provided so far. Graves et al. ${ }^{24}$ discussed a non-local transfer of the angular momentum in FeCoGd films between chemically different nanograins. More recently, Wienholdt et al. ${ }^{12}$ performed $a b$ initio calculations using DFT to develop an orbital-resolved model for spin dynamics in RETM alloys. From their model, they concluded that the ferromagnetic-like state observed by Radu et al. ${ }^{23}$ in FeCoGd is a consequence of 'dissipationless spin dynamics' during the first picoseconds after the excitation during which the energy and angular momentum is redistributed between the RE and TM elements. The experimental results presented here support this model.

In our work we measure $L^{i}(t), S^{i}(t)$ and $J^{i}(t)$ for each element and attribute the constant value of $J(t)$ in $\mathrm{Co}_{0.74} \mathrm{~Tb}_{0.26}$ during the first $140 \mathrm{fs}$ to the angular momentum flowing between $J^{\mathrm{Co}}(t)$ and $J^{\mathrm{Tb}}(t)$ (Fig. 4b, blue symbols). This process is hidden in $\mathrm{Co}_{0.8} \mathrm{Gd}_{0.2}$ because of the weak $J(t)$. This mechanism allows an ultrafast and local demagnetization in both sublattices, whereas it conserves the total angular momentum $J(t)$. We would like to point out that phonon- or defect-induced spin-flip scattering or superdiffusion of hot electrons would result in a decrease of $J(t)$ if measured by XMCD. According to our analysis, these mechanisms do not show a major contribution up to $140 \pm 60 \mathrm{fs}$. After $t=300 \mathrm{fs}$, the magnetization of Co reaches a minimum while the magnetization of Tb keeps decreasing, accompanied by a decrease of $J(t)$. In this case, the transfer channel of angular momenta between sublattices is no more efficient and angular momentum is transferred from the Tb sublattice towards an external reservoir (phonons, hot electrons and so on). The transfer rate towards the external reservoir is now set by the transfer rate from the $4 f$ electrons, which is usually slower than that for the $3 d$ electrons in TM elements. We thus have clearly demonstrated that an efficient transfer channel for angular momenta exists in multisublattice ferrimagnetic alloys before any transfer to the external bath. This two-step transfer mechanism agrees perfectly with the theoretical calculations performed by Wienholdt et al. ${ }^{12}$. Furthermore, our results show that this transfer results from a direct exchange of angular momenta between Co and $\mathrm{Tb}$ as long as both sublattices are demagnetizing ( $t<300 \mathrm{fs})$. In previous work by Radu et $a l^{23}$, uncorrelated demagnetization between the two sublattices was detected in the sub-picosecond timescale. Going one step further and considering the quantitative angular momenta $J^{i}(t)$, we show here that the two sublattices are strongly linked between $t=0$ and $140 \pm 60 \mathrm{fs}$ corresponding to the timescale of the exchange interaction 9 .

To conclude, our findings demonstrate the local transfer of angular momenta between the two antiferromagnetically exchange-coupled sublattices. This transfer channel induces ultrafast demagnetization at the atomic scale, whereas the total angular momentum $J(t)$ initially stays constant. In $\mathrm{Co}_{0.74} \mathrm{~Tb}_{0.26}$ we observed a delayed transfer of $J(t)$ to an external bath. These results evidence a new ultrafast mechanism, determine the related timescale for angular momentum transfer during the demagnetization in ferrimagnetic systems and are supported by recent $a b$ initio DFT calculations ${ }^{12}$. In addition, we have uncovered an ultrafast quenching of the $L^{\mathrm{Co}}(t) / S^{\mathrm{Co}}(t)$ ratio in ferrimagnetic $\mathrm{Co}_{0.8} \mathrm{Gd}_{0.2}$ and $\mathrm{Co}_{0.74} \mathrm{~Tb}_{0.26}$ alloys and attributed it to the ultrafast quenching of MCA in agreement with Bruno's model and previous results in $\mathrm{CoPd}^{10,30}$. For the RE elements, a different behaviour is observed for $L(t) / S(t)$ that could be linked to the localized character of the $4 f$ moments. 


\section{Methods}

Sample preparation and magnetic properties of the films. Fifteen nanometres thick Co-RE alloys have been grown by magnetron sputtering on $\mathrm{Si}_{3} \mathrm{~N}_{4}$ membranes. Co-deposition with convergent $\mathrm{Co}$ and $\mathrm{Gd}$ (or Tb) flux was used to get amorphous $\mathrm{Co}_{0.8} \mathrm{Gd}_{0.2}$ and $\mathrm{Co}_{0.74} \mathrm{~Tb}_{0.26}$ alloy films. The concentration in the films were optimized to obtain moderate saturation fields of $5 \mathrm{kOe}$ or less, compatible with the magnet used in the time-resolved experiments. For $\mathrm{Co}_{0.8} \mathrm{Gd}_{0.2}$ and $\mathrm{Co}_{0.74} \mathrm{~Tb}_{0.26}$ we have verified experimentally that at working temperatures the saturation fields are 4 and $3 \mathrm{kOe}$, respectively. Both alloys have been characterized by static $\mathrm{XMCD}^{27}$. As the samples were fully saturated by the external magnetic field of $5 \mathrm{kOe}$ during the pump-probe experiments, we can define $L_{\mathrm{z}}\left(\right.$ resp. $\left.S_{\mathrm{z}}\right)=L$ (resp. $S$ ) so that it follows that $J^{i}(t)=L^{i}(t)+S^{i}(t)$ (Fig. 4a, black and red symbols and Fig. $4 \mathrm{~b}$, black and red symbols) as the angular momentum of the element $i$ (Co, $\mathrm{Gd}$ or $\mathrm{Tb}$ ). The $\mathrm{Co}_{0.8} \mathrm{Gd}_{0.2}$ and $\mathrm{Co}_{0.74} \mathrm{~Tb}_{0.26}$ films show magnetic compensation temperatures $\left(T_{\text {comp }}\right)$ at which both magnetizations of the Co and the RE sublattices compensate (resp. $T_{\text {comp }}=150$ and $\left.550 \mathrm{~K}\right)$ and Curie temperatures $\left(T_{\mathrm{C}}\right)$ where the magnetic order is lost (resp. $T_{\mathrm{C}}=450$ and $650 \mathrm{~K}$ ).

The $\mathrm{Co}_{74} \mathrm{~Tb}_{26}$ alloy film presents an out-of-plane magnetic anisotropy, which is measured along the normal of the film plane. We emphasize that, in contrast to $\mathrm{CoPd}$ and $\mathrm{Co}_{0.74} \mathrm{~Tb}_{0.26}$ alloys ${ }^{10,27}, \mathrm{Co}_{0.80} \mathrm{Gd}_{0.20}$ has no out-of-plane magnetic anisotropy. For this sample we performed our XMCD experiment at $30^{\circ}$ from the normal of the film plane. In the case of $\mathrm{Co}_{0.80} \mathrm{Gd}_{0.20}$ an in-plane uniaxial anisotropy may be induced during growth ${ }^{32}$. This point is confirmed by our recent results where static XMCD characterization was performed ${ }^{27}$. In both alloys we found large ratios $L / S$ of $0.21-0.29$ at the Co $\mathrm{L}_{2,3}$ edges, whereas bulk Co shows a ratio of only 0.13 . This is a strong indication for the presence of large MCA energies in all our Co-RE compounds. However, in our alloys, the quantitative value of the spin momentum (for instance, $S^{\mathrm{Tb}}=1.35 \pm 0.2 \hbar \mathrm{at}^{-1}$ ) is lower than expected from Hund's rules $\left(S^{\mathrm{Tb}}=3 \hbar\right.$ at $\left.^{-1}\right)$ and is related to the structural disorder and finite temperature effects ${ }^{27}$.

Time-resolved XMCD. Time-resolved XMCD was performed at the femtoslicing beam line of the BESSY II synchrotron radiation source of the Helmholtz-Zentrum Berlin $^{7,10}$. The magnetization dynamics have been measured by monitoring the transmission signal of circularly polarized X-rays, tuned to specific core-level absorption edges as a function of a pump-probe delay. The dynamic XMCD contrast is obtained by subtracting the gated signals obtained with and without pump beam. The energy was set to the different $\mathrm{Co}_{2,3}, \mathrm{~Tb} \mathrm{M}_{4,5}$ and $\mathrm{Gd} \mathrm{M}_{4,5}$ edges using the Bragg Fresnel reflection zone plate monochromator. The experiments have been performed with a pump-probe set-up where the short X-ray pulses are synchronized with a femtosecond pump laser working at $790 \mathrm{~nm}, 3 \mathrm{kHz}$ repetition rate with pulses of $60 \mathrm{fs}$. The X-ray pulse duration of about $100 \mathrm{fs}$ in the femtoslicing operation mode ensures a global time resolution of $\sim 130 \mathrm{fs}$ (see refs 7,10 for details). The pump fluences used during our experiments were adjusted to $8 \mathrm{~mJ} \mathrm{~cm}{ }^{-2}$ for $\mathrm{Co}_{0.8} \mathrm{Gd}_{0.2}$ and to $12 \mathrm{~mJ} \mathrm{~cm}{ }^{-2}$ for $\mathrm{Co}_{0.74} \mathrm{~Tb}_{0.26}$ to reach large demagnetization magnitudes of about $60 \%$ at the $\mathrm{Co} \mathrm{L}_{3}$ edge without altering the sample properties (alloy concentration, atomic diffusion and large heating).

Fitting procedure. The physical quantities $L^{i}(t), S^{i}(t)$ and $J^{i}(t)(i=\mathrm{Co}, \mathrm{Gd}, \mathrm{Tb})$ were adjusted using the rate equation of the two-temperature model with two exponential functions (equation (1)):

$$
F(t)=G(t) \times\left\{C_{0}+C_{1} H\left(t-t_{0}\right)\left[1-\exp \left(-\left(t-t_{0}\right) / \tau_{\text {th }}\right)\right] \exp \left(-\left(t-t_{0}\right) / \tau_{\mathrm{s}-\mathrm{ph}}\right\}\right.
$$

where $G(t)$ is the Gaussian function defining the total time resolution of the experiment $(130 \mathrm{fs}), \tau_{\mathrm{th}}$ and $\tau_{\mathrm{s}-\mathrm{ph}}$ are the thermalization time and the relaxation time from the spin system to other systems (lattice, external bath), $t_{0}$ is the delay at which the temporal overlap of the pump and the probe is achieved and $H\left(t-t_{0}\right)$ is the Heaviside function $\left(H\left(t-t_{0}\right)=0\right.$ if $t<t_{0}$ and $H\left(t-t_{0}\right)=1$ if $\left.t>t_{0}\right)$ describing the energy transfer from the laser.

The parameters $C_{0}, C_{1}, t_{0}$ and $\tau_{\text {th }}$ in equation (1) were optimized to minimize independently the $\chi^{2}$ factor for $L^{i}(t), S^{i}(t)$ and $J^{i}(t)$ (equation (2)).

$$
\chi^{2}=\sum_{j}\left(\left(y-y_{j}\right) / \sigma_{j}\right)^{2}
$$

where $y$ is the rate equation, $y_{j}$ are the experimental data and $\sigma_{j}$ is the standard deviation (for $j=1-n$, and $n$ is the number of data points).

The minimization was performed via the Levenberg-Marquardt algorithm, based on a nonlinear least square procedure. The extracted error bars for $\tau_{\text {th }}$ and $t_{0}$ correspond to the standard deviation (s.d.), which is given as an output parameter of the fitting procedure.

The error bars appearing in Figs $2 \mathrm{~b}, \mathrm{c}$ and $3 \mathrm{~b}, \mathrm{c}$ for $L^{i}(t)$ and $S^{i}(t)$ as well as on Fig. $4 \mathrm{a}, \mathrm{b}$ for $f^{i}(t)$ correspond to the s.d. $(\sigma)$ of experimental data with respect to the fitting functions that minimize the $\chi^{2}$ factor. The amplitude of the error bars has been calculated according to equation (3):

$$
\sigma=\left(1 /(n-1) \times \sum\left(y-y_{j}\right)^{2}\right)^{1 / 2} .
$$

The final fitting parameters for $J^{i}(t)$ are compatible with the result of the sum of the two-fit functions obtained for $L^{i}(t)$ and $S^{i}(t)$.

The total angular momentum for each alloy was obtained by performing the weighted sum $J(t)=J^{\mathrm{Co}}(t)+J^{\mathrm{Tb}}(t)$ according to the composition of the alloy. In $\mathrm{Co}_{0.8} \mathrm{Gd}_{0.2}$, the total angular momentum $J^{\mathrm{Co}}(t)+J^{\mathrm{Gd}}(t)$ remains small and comparable to the noise level. For $\mathrm{Co}_{0.74} \mathrm{~Tb}_{0.26}$, a sizable value of $J(t)=J^{\mathrm{Co}}(t)+$ $J^{\mathrm{Tb}}(t)$ is obtained. In this case, we used the rate equation (1) to adjust $J(t)$ (blue solid line in Fig. 4). In parallel, we plotted the weighted sum-of-the-fit functions obtained for $J^{\mathrm{Co}}(t)$ and $J^{\mathrm{Tb}}(t)$; (magenta dotted line in Fig. 4). The two-fit functions for $J(t)$ agree perfectly for our parameters $C_{\mathrm{o}}, C_{1}, t_{0}$ and $\tau_{\mathrm{th}}$. The error bars given in Fig. 4a,b for $J(t)$ correspond to the s.d. of the fitting function derived using equation (3). The results extracted from the rate equation lead to the following zero-time values: $t_{0}=0 \pm 40 \mathrm{fs}$ for $J^{\mathrm{Co}}(t)$ and $J^{\mathrm{Tb}}(t)$ and $t^{\prime}{ }_{0}=140 \pm 40 \mathrm{fs}$ for $J(t)$, which allows us to define $\delta t=140 \pm 60 \mathrm{fs}$. The error bar for $\delta t$ has been derived as follows:

Since the main source of uncertainty for $\delta t$ stems from the error bars of $t_{0}$ as extracted from the rate equation, we performed the simulations using a time axis for $J^{\mathrm{Co}}(t)$ shifted by an amount of $\pm 40 \mathrm{fs}$ with respect to $J^{\mathrm{Tb}}(t)$. Doing so, we extracted the minimum and maximum delay times $\delta t$ for $J(t)$ of 80 and $200 \mathrm{fs}$. These lower and upper limits define our error bar of $60 \mathrm{fs}$ for the delais $\delta t=140 \mathrm{fs}$.

\section{References}

1. Beaurepaire, E., Merle, J. C., Daunois, A. \& Bigot, J.-Y. Ultrafast spin dynamics in ferromagnetic nickel. Phys. Rev. Lett. 76, 4250-4253 (1996).

2. Stanciu, C. D. et al. All-optical magnetic recording with circularly polarized light. Phys. Rev. Lett. 99, 047601 (2007).

3. Guidoni, L., Beaurepaire, E. \& Bigot, J.-Y. Magneto-optics in the ultrafast regime: thermalization of spin populations in ferromagnetic films. Phys. Rev. Lett. 89, 017401 (2002).

4. Wietstruk, M. et al. Hot-electron-driven enhancement of spin-lattice coupling in $\mathrm{Gd}$ and $\mathrm{Tb} 4 \mathrm{f}$ ferromagnets observed by femtosecond X-ray magnetic circular dichroism. Phys. Rev. Lett. 106, 127401 (2011)

5. Koopmans, B. et al. Explaining the paradoxical diversity of ultrafast laser induced demagnetization. Nat. Mater. 9, 259-265 (2010).

6. Zhang, G. P., Hübner, W., Lefkidis, G., Bai, Y. \& George, T. F. Paradigm of the time-resolved magneto-optical Kerr effect for femtosecond magnetism. Nat. Phys. 5, 499-502 (2009).

7. Stamm, C. et al. Femtosecond modification of electron localization and transfer of angular momentum in nickel. Nat. Mater. 6, 740-743 (2007).

8. Lopez-Flores, V. et al. Time-resolved $\mathrm{x}$-ray magnetic circular dichroism study of ultrafast demagnetization in a CoPd ferromagnetic film excited by circularly polarized laser pulse. Phys. Rev. B 86, 014424 (2012).

9. Stöhr, J. \& Siegmann, H. C. Magnetism: From Fundamentals to Nanoscale Dynamics (Springer, 2006).

10. Boeglin, C. et al. Distinguishing the ultrafast dynamics of spin and orbital moments in solids. Nature 465, 458-462 (2010).

11. Carpene, E. et al. Dynamics of electron-magnon interaction and ultrafast demagnetization in thin iron films. Phys. Rev. B 78, 174422 (2008).

12. Wienholdt, S., Hinzke, D., Carva, K., Oppeneer, P. M. \& Nowak, U. Orbitalresolved spin model for thermal magnetization switching in rare-earth-based ferrimagnets. Phys. Rev. B 88, 020406(R) (2013).

13. Carva, K., Battiato, M. \& Oppeneer, P. M. Ab Initio Investigation of the ElliottYafet electron-phonon mechanism in laser-induced ultrafast demagnetization. Phys. Rev. Lett. 107, 207201 (2011).

14. Battiato, M., Carva, K. \& Oppeneer, P. M. Supperdiffusive spin transport as a mechanism of ultrafast demagnetization. Phys. Rev. Lett. 105, 027203 (2010).

15. Malinowski, G. et al. Control of speed and efficiency of ultrafast demagnetization by direct transfer of spin angular momentum. Nat. Phys. 4, 855-888 (2008).

16. Battiato, M., Carva, K. \& Oppeneer, P. M. Theory of laser-induced ultrafast supperdiffusive spin transport in layered heterostructures. Phys. Rev. B 86, 024404 (2012).

17. Dalla Longa, F., Kohlhepp, J. T., de Jonge, W. J. M. \& Koopmans, B. Influence of photon angular momentum on ultrafast demagnetization in nickel. Phys. Rev. B 75, 224431 (2007).

18. Vodungbo, B. et al. Laser-induced ultrafast demagnetization in the presence of a nanoscale magnetic domain network. Nat. Commun. 3, 999 (2012).

19. Turgut, E. et al. Controlling the competition between optically induced ultrafast spin-flip scattering and spin transport in magnetic multilayers. Phys. Rev. Lett. 110, 197201 (2013).

20. Schellekens, A. J., Verhoeven, W., Vader, T. N. \& Koopmans, B. Investigating the contribution of superdiffusive transport to ultrafast demagnetization of ferromagnetic thin films. Appl. Phys. Lett. 102, 252408 (2013).

21. Schellekens, A. J. \& Koopmans, B. Microscopic model for ultrafast magnetization dynamics of multisublattice magnets. Phys. Rev. B 87, 020407 (2013).

22. Mentink, J. H. et al. Ultrafast spin dynamics in multisublattice magnets. Phys Rev. Lett. 108, 057202 (2012). 
23. Radu, I. et al. Transient ferromagnetic-like state mediated ultrafast reversal of antiferromagnetically coupled spins. Nature 472, 205-209 (2011).

24. Graves, C. E. et al. Nanoscale spin reversal by non-local angular momentum transfer following ultrafast laser excitation in ferrimagnetic GdFeCo. Nat. Mater. 35, 293-298 (2013).

25. Thole, B. T., Carra, P., Sette, S. \& van der Laan, G. X-ray circular dichroism as a probe of orbital magnetization. Phys. Rev. Lett. 68, 1943-1946 (1992).

26. Carra, P., Thole, B. T., Altarelli, M. \& Wang, X. X-ray circular dichroism and local magnetic fields. Phys. Rev. Lett. 70, 694-697 (1993).

27. Lopez-Flores, V. et al. Role of critical spin fluctuations in ultrafast demagnetization of transition-metal rare-earth alloys. Phys. Rev. B 87, 214412 (2013).

28. Koopmans, B., Kicken, H. H. J. E., van Kampen, M. \& de Jonge, W. J. M. Microscopic model for femtosecond magnetization dynamics. J Magn. Magn. Mater. 286, 271-275 (2005).

29. Teramura, Y., Tanaka, A., Thole, B. \& Jo, T. Effect of Coulomb interaction on the $\mathrm{X}$-ray magnetic circular dichroism spin sum rule in $3 \mathrm{~d}$ transition elements. J. Phys. Soc. Jpn 65, 1053-1055 (1996).

30. Bruno, P. Tight-binding approach to the orbital magnetic moment and magnetocrystalline anisotropy of transition-metal monolayers. Phys. Rev. B 39, 865-868 (1989).

31. Medapalli, P. et al. Efficiency of ultrafast laser-induced demagnetization in $\mathrm{Gd}_{x} \mathrm{Fe}_{100-x-y} \mathrm{Co}_{y}$ alloys. Phys. Rev. B 86, 054442 (2012).

32. Dufour, C., Dumesnil, K. \& Mangin, P. H. Strain-induced modification of magnetic structure and new magnetic phases in rare-earth epitaxial films. PRAMANA 67, 173-190 (2006).

\section{Acknowledgements}

We are indebted to A Eschenlohr, K Holldack, R Mitzner and T Kachel for help and support during the femtoslicing experiments, and A Boeglin for discussions and correction of the manuscript. This work was supported by the CNRS-PICS, by Universite de Strasbourg and the EU Contract Integrated Infrastructure Initiative I3 in FP6-Project number R II 3 CT-2004-5060008, BESSY IA-SFS Access Program, by the 'Agence Nationale de la Recherche' in France via the project EQUIPEX UNION: number ANR10-EQPX-52' and by the German Ministry of Education and Research BMBF Grant 05K10PG2 (FEMTOSPEX). V.L.-F. acknowledges the Ministry of Education of Spain (Programa Nacional de Movilidad de Recursos Humanos del Plan Nacional de I-D +i 2008-2011) for financial support.

\section{Author contributions}

N.B., V.L.-F., V.H., N.P., C.S, E.B. and C.B. performed the time-resolved measurements and data exploitations. M.H. grew and characterized the samples. N.B. and C.B. wrote the paper. All authors discussed and improved the manuscript.

\section{Additional information}

Competing financial interests: The authors declare no competing financial interests.

Reprints and permission information is available online at http://npg.nature.com/ reprintsandpermissions/

How to cite this article: Bergeard, N. et al. Ultrafast angular momentum transfer in multisublattice ferrimagnets. Nat. Commun. 5:3466 doi: 10.1038/ncomms4466 (2014). 